\title{
Review of: "Post COVID-19 Guillain Barre Syndrome in Immunocompromised Children - A report of two cases and review of literature"
}

\author{
Alessandro Introna
}

Potential competing interests: The author(s) declared that no potential competing interests exist.

The paper deals with two cases of pediatric Guillain-Barré syndrome (GBS) interpreted by the authors as consequence of SARS-CoV-2 infection. As stated by the authors, data regarding pediatric GBS related to SARS-CoV-2 infection are limited.

Nonetheless, there are some concerns about the paper:

- first of all, a recent international prospective cohort study ${ }^{[1]}$ suggests that a strong association between SARS-CoV-2 and GBS is unlikely considering that there was not an increase in inclusion rate of the International GBS Outcome Study (IGOS). Nevertheless, the authors did not exclude that SARS-CoV-2 infection could trigger GBS. GBS in the context of confirmed or probable preceding SARS-CoV-2 infection shared common features. First of all, the sensorimotor and demyelinating features were predominant with frequent involvement of facial nerves. Herein, the patients presented pure motor variant, with no cranial nerves involvement in the first case and "generic" bulbar involvement in the second. The authors should discuss the different neurophysiological presentation of their cases and present extensively the contrasting literature data on association of COVID-19 and GBS.

- another important point is the criteria used to define GBS related to SARS-CoV-2 infection. Ellul et al $^{[2]}$ proposed case definition for Guillain-Barré syndrome associated with SARS-CoV-2 infection. Probable association requires: 1) Neurological disease onset within 6 weeks of acute infection; and (2) either SARS-CoV-2 RNA detected in any sample or antibody evidence of acute SARS-CoV-2 infection; and (3) no evidence of other commonly associated causes. In the first case the patient had respiratory symptoms two weeks before the onset of neurological symptoms and RT-PCR tested positive for COVID19. In the second case, the patient tested positive for SARS-CoV2 antibodies (without mention about acute infection) and there was no clue of precedent respiratory syndrome. Both cases did not rule out other commonly associated causes of GBS. The authors cited Dalakas et al ${ }^{[3]}$ suggesting that many GBS patients do not have any COVID-19 symptoms. The diagnosis should be especially suspected in cases with anosmia, ageusia, cranial neuropathies, or lymphocytopenia. The second case did not present with none of these symptoms. I suggest addressing these issues.

- Lastly, in my opinion another point of discussion is the treatment of patient with poor outcome. As the authors suggested in the additional comments of Table, the second patient needed for a second course of endovenous immunoglobulin because of symptoms worsening. Recently, the results of SID-GBS 
(Second intravenous immunoglobulin dose in patients with Guillain-Barré syndrome with poor prognosis ${ }^{[4]}$ shed light on the potential benefit of a second course of immunoglobulin dose in patients with poor prognosis GBS. The study did not provide evidence of benefit for a second course entailing severe adverse events.

\section{References}

1. `Linda W G Luijten, Sonja E Leonhard, Annemiek A van der Eijk, Alex Y Doets, et al. (2021). GuillainBarré syndrome after SARS-CoV-2 infection in an international prospective cohort study. doi:10.1093/brain/awab279.

2. ^Mark A Ellul, Laura Benjamin, Bhagteshwar Singh, Suzannah Lant, et al. (2020). Neurological associations of COVID-19. The Lancet Neurology, vol. 19 (9), 767-783. doi:10.1016/s14744422(20)30221-0.

3. `Marinos C. Dalakas. (2020). Guillain-Barré syndrome: The first documented COVID-19-triggered autoimmune neurologic disease. Neurol Neuroimmunol Neuroinflamm, vol. 7 (5), e781. doi:10.1212/nxi.0000000000000781.

4. `Christa Walgaard, Bart C Jacobs, Hester F Lingsma, Ewout W Steyerberg, et al. (2021). Second intravenous immunoglobulin dose in patients with Guillain-Barré syndrome with poor prognosis (SIDGBS): a double-blind, randomised, placebo-controlled trial. The Lancet Neurology, vol. 20 (4), 275-283. doi:10.1016/s1474-4422(20)30494-4. 